\title{
Validation of a Score Developed to Estimate the 6-month Survival of Patients Treated with Palliative Local Radiotherapy for Advanced Lung Cancer
}

\author{
STEFAN JANSSEN ${ }^{1}$, BIRGITT VAN OORSCHOT $^{2}$, LUKAS KÄSMANN ${ }^{1}$, STEVEN E. SCHILD $^{3}$ and DIRK RADES ${ }^{1}$ \\ ${ }^{1}$ Department of Radiation Oncology, University of Lübeck, Lübeck, Germany; \\ ${ }^{2}$ Department of Radiation Oncology, University of Würzburg, Würzburg, Germany; \\ ${ }^{3}$ Department of Radiation Oncology, Mayo Clinic, Scottsdale, AZ, U.S.A.
}

\begin{abstract}
Aim: To validate a score estimating the 6-month survival of lung cancer patients irradiated for locally advanced disease. Patients and Methods: The validation cohort of this study included 125 patients. The score for each patient was calculated by adding the points (6-month survival divided by 10) from the factors lymph node stage, distant metastasis and performance score. Patients were divided into three groups, 10-11 (N=36), 12-14 $(N=69)$ and 15-17 points $(N=20)$. Six-month survival of each group from the original study was compared to its corresponding group from this validation study. Results: Six-month survival rates were $19 \%$ (10-11 points), $51 \%$ (12-14 points) and $75 \%$ (1617 points) in the validation cohort $(p<0.001)$ and $13 \%, 47 \%$ and $82 \%$ in the previous study $(p<0.001)$. The group-bygroup comparisons between both cohorts did not reveal any significant differences. Conclusion: The score was reproducible and valid for estimating the 6-month survival of lung cancer patients assigned to palliative thoracic radiotherapy.
\end{abstract}

Many patients with locally advanced lung cancer are not able to receive curative treatment, such as thoracic surgery or chemo-radiation with radiation doses $\geq 60$ Gy $(1,2)$. These patients are often assigned to receive palliative thoracic radiotherapy, mainly to control or prevent cancer-related clinical symptoms (3). Since many of these patients present with poor performance status and significant co-morbidity, the regimen used for palliative radiotherapy should be

Correspondence to: Dirk Rades, MD, Department of Radiation Oncology, University of Lübeck, Lübeck, Ratzeburger Allee 160, D-23562 Lübeck, Germany. Tel: +49 45150045401, Fax: +49 45150045404, e-mail: Rades.Dirk@gmx.net

Key Words: Locally advanced lung cancer, palliative radiotherapy, survival prognosis, prognostic score, validation, thoracic radiotherapy. tailored to individual patients taking into account the patients' survival prognosis (4). In patients with very poor prognoses, the overall treatment time should be kept reasonably short so that patients avoid spending their short remaining lifetime receiving treatment.

On the other hand, patients with a more favorable survival prognosis may benefit from a longer-lasting radiotherapy regimen with lower doses per fraction. Higher doses are associated with more prolonged local control and lower doses per fraction are generally associated with a lower risk of late radiation-related toxicity $(5,6)$. The risk of late toxicity increases with a patient's lifetime. Thus, it is very helpful to be able to estimate a patient's survival prognosis prior to the start of palliative radiotherapy to help one best match the most appropriate course of therapy to the needs of the patient. To facilitate this estimation, we performed a study in 125 lung cancer patients receiving palliative local radiotherapy and created a survival score particularly for this group (7). At that time, we were unable to validate the score due to a lack of patients. Recently, a group from Norway aimed to validate our score in a retrospective cohort treated at their Institution (8). However, the authors compared their cohort to the cohort of our previous study for median survival times and 1-year survival rates but not for 6-month survival rates, the end-point our score was designed for (7). Therefore, the present study was performed in order to validate the 6-month survival score with a study including 125 new patients.

\section{Patients and Methods}

The cohort of this retrospective study represented the validation group for our prognostic instrument that was developed to specifically estimate the 6-month survival probability of lung cancer patients treated with palliative thoracic radiotherapy for locally advanced disease. This validation cohort included 125 patients, 104 patients from a previous study of the University of Würzburg (9) and 21 new patients (i.e. not included in the previous study) from 
our Institution, the University of Lübeck. Thoracic radiotherapy was performed with a modern linear accelerator after computed tomography-based three-dimensional treatment planning. Planned radiation doses (equivalent doses in 2 Gy fractions) ranged between 32.5 and 56 Gy (median dose=42.3 Gy) (5).

The prognostic score for each patient was calculated by adding the scoring points from the three factors that formed the basis of the previously created survival score, namely lymph node stage (N0-1 versus N2-3), distant metastasis (M0 versus $\mathrm{M} 1$ ) and the performance status given by the Karnofsky performance score (KPS $\leq 60 \%$ versus $\mathrm{KPS} \geq 70 \%$ ). The 6 -month survival rates, based on each of these three factors, were divided by 10 (Table I). The sum of the point values obtained for the three factors represented the total score for each patient. The total scores in the validation cohort also ranged between 10 and 17 points and patients were divided into the same three groups, $10-11$ points $(\mathrm{N}=36), 12-14$ points $(\mathrm{N}=69)$ and $15-17$ points $(\mathrm{N}=20)$. These groups were compared for survival using the Kaplan-Meier method and the Wilcoxon test (10).

In order to validate the previously developed score, each group of the original study (10-11 points, $12-14$ points, $15-17$ points) was compared to its corresponding group of the present validation study with respect to the 6-month survival rates using the chi-square test.

\section{Results}

In the current validation study, the 6-month survival rates were $19 \%$ in the $10-11$ points group, $51 \%$ in the $12-14$ points group and $75 \%$ in the $16-17$ points group, respectively (Figure 1, $<<0.001$ ). The difference in 6-month survival between the $10-11$ points and the $12-14$ points group was significant $(p=0.016)$, whereas the difference between the 12-14 points and the 16-17 points group was not $(p=0.20)$.

The corresponding 6-month survival rates in the previous study were $13 \%$ in the $10-11$ points group, $47 \%$ in the $12-14$ points group and $82 \%$ in the $16-17$ points group, respectively $(p<0.001)$. The group-by-group comparisons between the cohort from the previous study and the cohort of the current validation study did not reveal any significant differences. The p-values were 0.49 for the comparison of both $10-11$ points groups, 0.74 for the comparison of both 12-14 points groups and 0.79 for the comparison of both 15-17 points groups, respectively.

The score was specifically designed to estimate the 6-month survival probability of lung cancer patients irradiated locally with palliative intent. However, additional survival data in the present validation study were as follows: The 3-month survival rates in the three prognostic groups were $39 \%, 70 \%$ and $85 \%$, respectively. The 12-month survival rates were $14 \%, 27 \%$ and $38 \%$, respectively, whereas the median survival times were 2 months, 7 months and 9.5 months, respectively.

\section{Discussion}

The survival times of patients assigned to palliative thoracic radiotherapy of locally advanced lung cancer may vary considerably, ranging between a few weeks and a year or longer $(7,8)$. Personalization of palliative irradiation for lung cancer includes individualizing treatment to the patient's remaining lifespan $(11,12)$. In patients with a very poor survival prognosis, a short course of radiotherapy is more appropriate. One must avoid having these patients spending a substantial proportion of their limited remaining time with receiving treatment. Furthermore, many of these patients will likely not live long enough to experience late radiation-related toxicity that would be less pronounced if longer-course radiotherapy programs with lower doses per fraction (6). On the other hand, patients with a more favorable survival prognosis appear better treated with longer-course radiotherapy programs, including higher total doses and lower doses per fraction. Due to their longer remaining lifespan, these patients are at a greater risk of developing late toxicities. Moreover, in other palliative situations requiring radiotherapy, such as brain metastases and malignant epidural spinal cord compression, longer-course radiotherapy programs with higher total doses resulted in longer local control and survival than shorter programs with lower total doses $(13,14)$.

These considerations have led us to the development of this scoring system to estimate the 6-month survival probability of lung cancer patients treated with palliative radiotherapy for locally advanced disease (7). The scoring system included three prognostic groups with significantly different 6-month survival rates of $13 \%, 47 \%$ and $82 \%$, respectively $(p<0.001)$. However, this scoring system had not yet been properly validated. Recently, a group from Norway aimed to perform an external validation of our scoring system in a retrospective series of 232 lung cancer patients (8). The authors reported median survival times for the three prognostic groups of 1.2 months (10-11 points), 5.3 months (12-14 points) and 8.2 months (15-17 points). In the first two groups, the median survival times were similar to the 2 months and 6 months found in our previous study, in which the scoring system was developed. However, in 15-17 points group, the median survival times were quite different, i.e. 8.2 months in the series from Norway and 38 months in our previous study. In the Norwegian study, the 1-year survival rates of the three prognostic groups were $2 \%, 21 \%$ and $32 \%$, respectively. When compared to our previous study, the results were similar in the $10-11$ points group $(2 \%$ versus $8 \%$ ) and the $12-14$ points group (21\% versus $19 \%)$ but not in the $15-17$ points group $(32 \%$ versus $69 \%)(7,8)$. However, one has to keep in mind that our scoring system was designed specifically to estimate the 6-month survival probability, not the 1-year survival probability or the median survival time (7). We feel that the 6-month survival time is a reasonable cut-off for patients requiring palliative radiotherapy to ultimately help one individualize treatment. A similar cut-off is widely used for scoring systems developed to predict the survival of lung cancer patients receiving palliative radiotherapy of brain metastases or malignant epidural spinal cord compression $(15,16)$. 
Table I. Scoring points of the three factors included in the score (7).

\begin{tabular}{ll}
\hline Prognostic factor & Scoring points \\
\hline Karnofsky performance score & \\
$\leq 60$ & 3 \\
$\geq 70$ & 5 \\
Lymph node stage & \\
N0-N1 & 7 \\
N2-N3 & 3 \\
Distant metastasis & \\
M0 & 5 \\
M1 & 4 \\
\hline
\end{tabular}

Therefore, the attempt to validate our scoring system in the study from Norway did not investigate the specific end-point the scoring system was designed to predict $(7,8)$. However, they have validated that the score predicts survival.

The present validation study focused on the end-point the scoring system was specifically designed to predict, the 6month survival probability. A separate group-to-group comparison was performed between the test cohort of our previous study and the present validation cohort for each of the three prognostic groups 10-11 points, $12-14$ points and 15-17 points. The 6-month survival rates in both cohorts were similar between the cohorts and the group-to-group comparisons did not reveal a significant difference. Therefore, our scoring system appears, in principle, valid and reproducible with respect to estimating the 6-month survival probability of lung cancer patients treated with palliative thoracic radiotherapy.

One has to be aware that both of our studies were retrospective in nature and are, therefore, at risk of including hidden selection biases. Furthermore, the 12-14 points group and the 15-17 points group were less distinct in this validation study than in the original study. The absolute differences in 6month survival were $24 \%$ and $35 \%$, respectively. In the present study, the difference was not significant. Thus, the scoring system may not be sufficiently robust to appropriately differentiate between these two groups. In contrast, the absolute differences in 6-month survival between the 10-11 points and the $12-14$ points group were similar, i.e. $34 \%$ in the original study and $32 \%$ in this validation study (7). In this study, the difference between the 10-11 points and the 12-14 points group was significant. Thus, the scoring system appears suitable to identify patients with a poor 6-month survival probability, who would be candidates for short-course radiotherapy. When assigning a patient to a radiotherapy program, those groups with scores of 12-14 points and 15-17 points may be considered as one group. They could benefit from a longer-course radiotherapy program with a higher total dose and lower doses per fraction $(13,14)$.

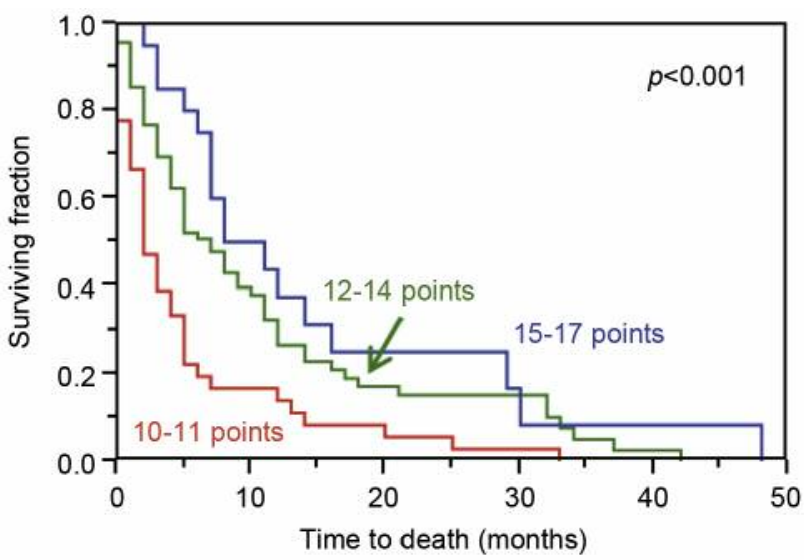

Figure 1. Kaplan-Meier curves of the three prognostic groups of the validation cohort.

In conclusion, according to the results of this validation study, our scoring system appears reproducible regarding its ability to estimate the 6-month survival probability of lung cancer patients assigned to local palliative radiotherapy. However, the difference between the prognostic groups of 12-14 and 15-17 points was less pronounced in the validation group than in the original study. Therefore, the scoring system may not appropriately differentiate between patients with an intermediate and those with a very favorable survival prognosis. The scoring system appears robust when it is used to identify patients with a poor 6-month survival probability.

\section{Conflicts of Interest}

On behalf of all Authors, the corresponding Author states that there is no conflict of interest related to this study.

\section{References}

1 Al-Shamsi HO, Al Farsi A and Ellis PM: Stage III non-smallcell lung cancer: Establishing a benchmark for the proportion of patients suitable for radical treatment. Clin Lung Cancer 15: 274-280, 2014.

2 Jeremic B, Shibamoto Y, Milicic B, Dagovic A, Nikolic N, Aleksandrovic J, Acimovic L and Milisavljevic S: Impact of treatment interruptions due to toxicity on outcome of patients with early stage (I/II) non-small-cell lung cancer (NSCLC) treated with hyperfractionated radiation therapy alone. Lung Cancer 40: 317-323, 2003.

3 Rodrigues G, Macbeth F, Burmeister B, Kelly KL, Bezjak A, Langer C, Hahn C and Movsas B: Consensus statement on palliative lung radiotherapy: Third international consensus workshop on palliative radiotherapy and symptom control. Clin Lung Cancer 13: 1-5, 2012.

4 Jeremic B, Fidarova E, Sharma V, Faheem M, Ameira AA, Nasr Ben Ammar C, Frobe A, Lau F, Brincat S and Jones G: The International Atomic Energy Agency (IAEA) randomized trial of palliative treatment of incurable locally advanced non small cell 
lung cancer (NSCLC) using radiotherapy (RT) and chemotherapy (CHT) in limited resource setting. Radiother Oncol 116: 21-26, 2015.

5 Joiner MC and Van der Kogel AJ: The linear-quadratic approach to fractionation and calculation of isoeffect relationships. In Steel GG (ed.): Basic clinical radiobiology. Oxford University Press New York, 106-112, 1997.

6 Barendsen GW: Dose fractionation, dose rate and iso-effect relationships for normal tissue responses. Int J Radiat Oncol Biol Phys 8: 1981-1997, 1982.

7 Rades D, Käsmann L, Schild SE and Janssen S: A survival score for patients receiving palliative irradiation for locally advanced lung cancer. Clin Lung Cancer 17: 558-562, 2016.

8 Nieder C, Tollali T, Haukland E, Reigstad A, Flatøy LR and Dalhaug A: External validation of a prognostic score for patients receiving palliative thoracic radiotherapy for lung cancer. Clin Lung Cancer pii: S1525-7304(17)30021-9, 2017. doi: 10.1016/j.cllc.2017.01.006. [Epub ahead of print]

9 Van Oorschot B, Assenbrunner B, Schuler M, Beckmann G and Flentje M: Survival and prognostic factors after moderately hypofractionated palliative thoracic radiotherapy for non-small cell lung cancer. Strahlenther Onkol 190: 270-275, 2014.

10 Kaplan EL and Meier P: Non-parametric estimation from incomplete observations. J Am Stat Assoc 53: 457-481, 1958.

11 Rades D and Schild SE: Lung cancer: Best supportive care - a reasonable option for patients with brain metastases? Nat Rev Clin Oncol 13: 722-724, 2016.
12 Janssen S, Kaesmann L, Schild SE and Rades D: Impact of the radiation dose and completion of palliative radiotherapy on survival in patients treated for locally advanced lung cancer. Anticancer Res 36: 1825-1828, 2016.

13 Rades D, Panzner A, Dziggel L, Haatanen T, Lohynska R and Schild SE: Dose-escalation of whole-brain radiotherapy for brain metastasis in patients with a favorable survival prognosis. Cancer 118: 3853-3859, 2012.

14 Rades D, Panzner A, Rudat V, Karstens JH and Schild SE: Dose escalation of radiotherapy for metastatic spinal cord compression (MSCC) in patients with relatively favorable survival prognosis. Strahlenther Onkol 187: 729-735, 2011.

15 Rades D, Douglas S, Veninga T and Schild SE: A validated survival score for patients with metastatic spinal cord compression from non-small cell lung cancer. BMC Cancer 12: 302, 2012.

16 Rades D, Dziggel L, Segedin B, Oblak I, Nagy V, Marita A, Schild SE, Trang NT and Khoa MT: A new survival score for patients with brain metastases from non-small cell lung cancer. Strahlenther Onkol 189: 777-781, 2013.
Received March 21, 2017

Revised April 1, 2017

Accepted April 3, 2017 